\title{
Aging in Dense Colloids as Diffusion in the Logarithm of Time
}

\author{
Stefan Boettcher ${ }^{1, *}$ and Paolo Sibani ${ }^{2, \text {, }}$ \\ ${ }^{1}$ Department of Physics, Emory University, Atlanta, GA, USA \\ ${ }^{2}$ Institut for Fysik og Kemi, SDU, DK5230 Odense M, Denmark
}

\begin{abstract}
The far-from-equilibrium dynamics of glassy systems share important phenomenological traits. A transition is generally observed from a time-homogeneous dynamical regime to an aging regime where physical changes occur intermittently and, on average, at a decreasing rate. It has been suggested that a global change of the independent time variable to its logarithm may render the aging dynamics homogeneous: for colloids, this entails diffusion but on a logarithmic time scale. Our novel analysis of experimental colloid data confirms that the mean square displacement grows linearly in time at low densities and shows that it grows linearly in the logarithm of time at high densities. Correspondingly, pairs of particles initially in close contact survive as pairs with a probability which decays exponentially in either time or its logarithm. The form of the Probability Density Function of the displacements shows that long-ranged spatial correlations are very long-lived in dense colloids. A phenomenological stochastic model is then introduced which relies on the growth and collapse of strongly correlated clusters ("dynamic heterogeneity"), and which reproduces the full spectrum of observed colloidal behaviors depending on the form assumed for the probability that a cluster collapses during a Monte Carlo update. In the limit where large clusters dominate, the collapse rate is $\propto 1 / t$, implying a homogeneous, log-Poissonian process that qualitatively reproduces the experimental results for dense colloids. Finally an analytical toy-model is discussed to elucidate the strong dependence of the simulation results on the integrability (or lack thereof) of the cluster collapse probability function.
\end{abstract}

PACS numbers:

\section{INTRODUCTION}

Aging in amorphous materials has attracted widespread experimental, simulational and theoretical interest for more than thirty years [1 [6]. As a spontaneous off-equilibrium relaxation process, aging entails a decrease of the free energy and, correspondingly, a slow change of thermodynamic averages. E. g., in numerical studies of models for disordered magnets, the thermal energy decreases intermittently and, on average, at a decelerating rate during the aging process $6-9]$. Like these low-temperature materials, colloidal suspensions at high density (i. e. high volume fraction) exhibit intermittent dynamics and a gradual slowing down, here, in the rate at which particles move during light scattering [10, 11] and particle tracking experiments $[12-15]$. While the phenomenology of colloidal aging is broadly similar to that of thermally activated aging, spatially averaged quantities as energy and particle density hardly change in colloids. Furthermore, no external field is required to elicit a measurable response. Finally, the motion of colloidal particles is time-homogeneous for sufficiently low density, but for no accessible time scales and for no value of the density does it look stationary or equilibrium-like. Generalizations of the Fluctuation Dissipation Theorem and the concept of effective temperature [16, 17], developed to describe thermally controlled aging in disordered magnets, are

*www.physics.emory.edu/faculty/boettcher/

tpaolo.sibani@ifk.sdu.dk not applicable to colloids, since the dichotomy between equilibrium-like fluctuations and off-equilibrium dynamics is absent. Reconciling differences and similarities between aging in colloids and other glassy systems thus requires a novel and broader approach .

In this work, the colloidal particle tracking experiments of Courtland et al. 13] are re-analyzed, leading to the conclusion that particle motion is diffusive in either time or logarithmic time, at low or high densities, respectively. Dynamics properties, such as the Mean Squared Displacement (MSD) and the persistence of tracer particles, appear homogeneous when viewed as a function of an appropriately re-scaled time variable. As the MSD, for instance, is a sum of independent displacements, the correct choice of time variable, hence, trivializes colloidal dynamics[30]. To explain why that is, a novel stochastic model is introduced with a single free parameter controlling the transition from diffusive to log-diffusive behavior. In the log-diffusive regime, the dynamics is driven by increasingly rare events, called "quakes", whose temporal distribution is homogeneous in logarithmic time. This links the dynamical behavior of dense colloids to the notion of "record dynamics" [18, 19], a relaxation paradigm already applied to a number of complex systems [3, 9, 20, 21].

Our experimental data analysis considers the MSD and the persistence, i. e. the probability that a pair of particles initially close to each other remain close for at least time $t$, under a number of different conditions. The Probability Density Function (PDF) of the particle displacement is likewise measured to illustrate intermittency and the long range of the spatial correlations. The model encodes the spatial heterogeneity in particle mobility, 

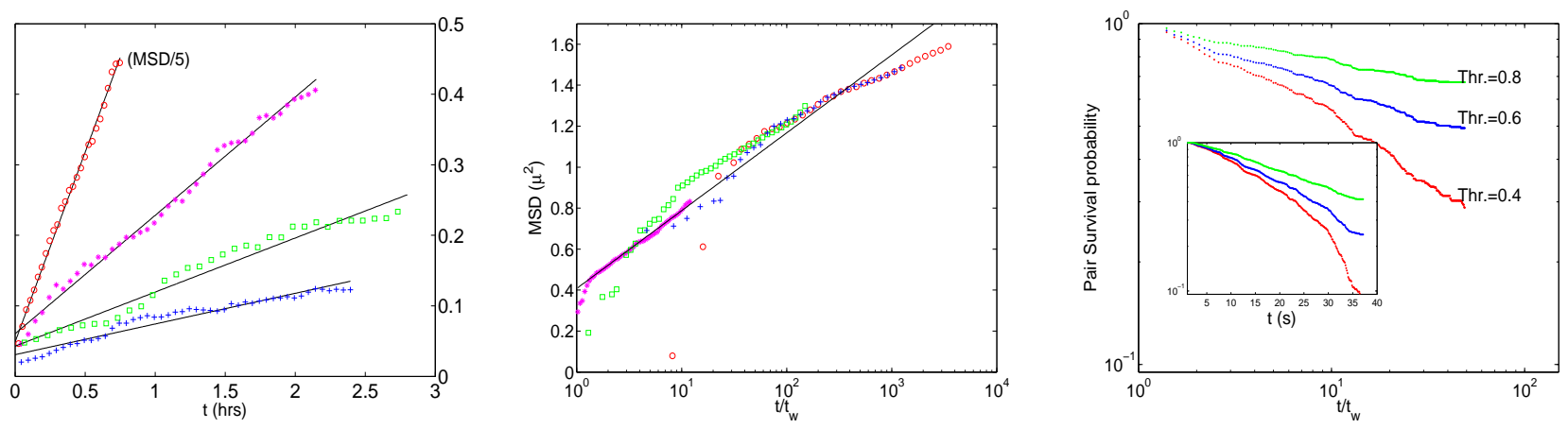

Figure 1: (Color online) Left: Mean Square Displacement (in $\mu^{2}$ ) for colloids at low density, volume fractions $\rho \approx$ $0.46,0.52,0.53,0.56$, top to bottom, all plotted versus time, in seconds. The top-most data set has, for graphical reasons, been scaled down by a factor of five. Middle: same quantity for dense colloids, where for all data $\rho \approx 0.62$. Waiting times, in seconds: $t_{w} \approx 1,10,20$, and 150 for pluses, squares, circles, and stars, respectively. Right: At high density, $(\rho \approx 0.62)$ the persistence decays as a power law dependent on a separation-threshold $\theta$. The inset shows the exponential decay at low density, $\rho \approx 0.46$, for the same thresholds.

which is ubiquitous in glassy systems [12, 15, 22, 23], in terms of the formation and evolution of clusters of strongly correlated spatially contiguous particles. These clusters signal emerging "dynamic heterogeneities" (DH), i. e. a broad distribution of time-scales for the survival and break-up of correlation patterns in different regions [12]. The stochastic model abstracts the underlying dynamics solely into a cluster survival probability: complex behavior replaces simple diffusive behavior once clusters attain non-zero probability to survive indefinitely under the update rule. When the probability per update to break up falls off exponentially with cluster size, events in the model become well-separated in time, with a log-Poissonian statistic. This reproduces the qualitative features of the experimental data: the dynamics is intermittent and homogeneous in logarithmic time.

\section{EXPERIMENTAL DATA ANALYSIS}

Obtained using particle tracking techniques, the colloidal data of Courtland et al. [13] comprise the $3 d-$ trajectories of a set of tagged colloidal particles. The particle radius is 1.18 microns, and the position coordinates are given in microns, a length unit nearly equal to the particle radius. Colloids were initially centrifuged to obtain the desired density, and then briefly stirred. The end of the stirring phase is considered as the origin of the time axis, i.e., $t=0$. Without time-translational invariance, this choice impacts the description of dense colloids.

The MSD, relative to any overall drift in the system, is calculated from the trajectories as a function of time. Fig. 11 leftmost panel, shows the well-known diffusive behavior of low density colloids. The diffusion coefficient decreases monotonically with increasing volume fraction. MSD pertaining to high-density colloids are plotted in the middle panel on a log horizontal scale versus the scal- ing variable $t / t_{w}$, where $t_{w}, t_{w} \leq t$ is the age at which data collection commences. Although a more precise knowledge of $t_{w}$ would facilitate a better data collapse, it is apparent that the particles in a dense colloid clearly diffuse in logarithmic time.

The rightmost panel of Fig. 1 displays data for the persistence defined above. To ensure statistical independence, we partition the system in $20 \times 20 \times 5$ identical sub-volumes. At time $t_{w}$, a particle centrally located in each sub-volume and its closest neighbor are tagged as a pair. If at time $t>t_{w}$ the distance between the elements of a pair increases beyond a given threshold value $\theta$ and subsequently remains above $\theta$, the pair is deemed to have broken up at $t$. In the plot, three $\theta$-values are considered. Measured in units of particle radius, these $\theta$ are large compared to the typical range of the relative motion. The persistence decays as a power of $t$ for dense colloids, i. e. exponentially in $\log (t)$, while at low density it relaxes exponentially, as shown in the insert.

Finally, Fig. 2 shows the empirical PDF (also known as the self-part of the Van Hove distribution function) of the absolute value of the particle displacement (in any direction) in the liquid (left panel) and glassy (right panel) regimes of the colloids. The vertical scale is logarithmic. The three data sets displayed correspond to different values of the time interval $\Delta t$ over which the displacement is observed. The inserts include more values of $\Delta t$, and merely confirm the diffusive, respectively log-diffusive, character of the dynamics previously discussed. Note that the MSD does not cross the origin. The particles already have acquired some dispersion after the first sweep of the confocal microscope, probably due to a residual stirring motion.

In similar analyses performed on a number of different systems [24, 25], a central Gaussian part is generally flanked by an exponential 'non-Fickian' tail. The left-most part of the PDF should, by analogy, have a parabolic shape, and some curvature is indeed visible in 

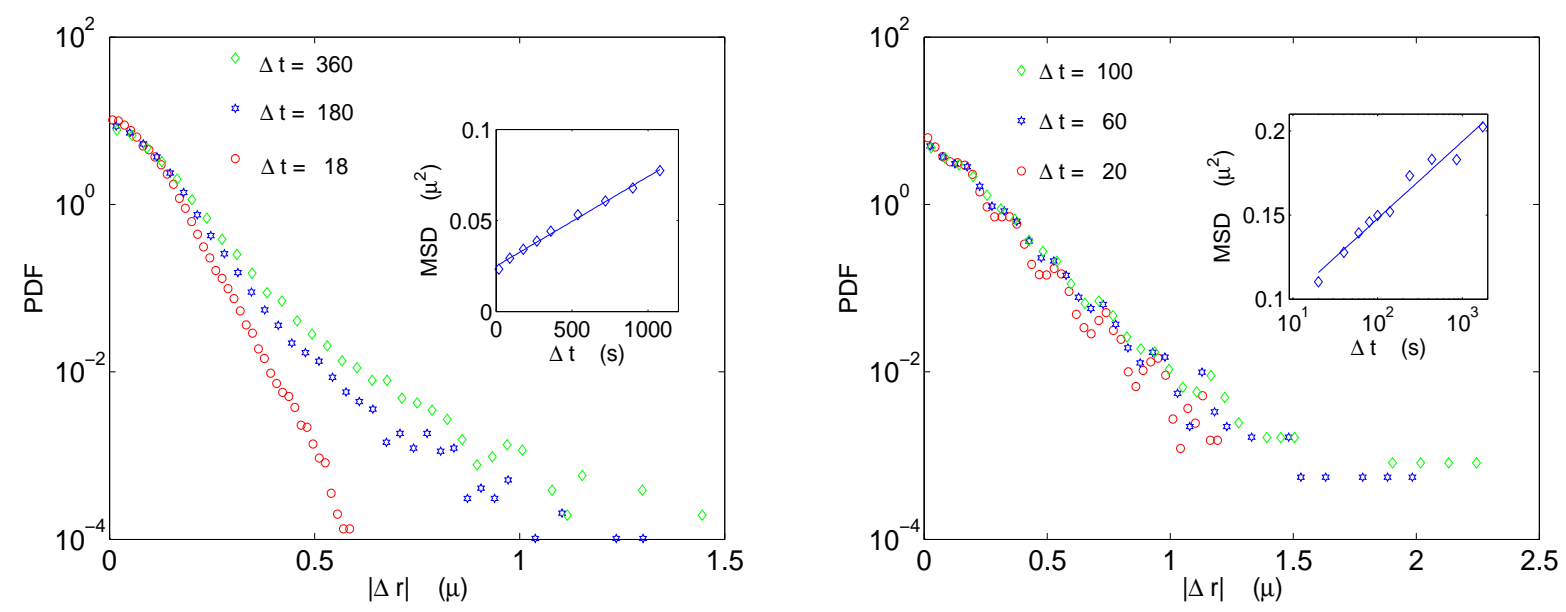

Figure 2: (Color online) The left panel pertains to the liquid, and the right one to the glassy regime of a colloid. Both panels display on a log-scale the PDF for the absolute value $|\Delta r|$ of the particle displacement (in any direction). Three data sets are shown, corresponding to different durations (in seconds) of the time interval $\Delta t$ over which the displacement is measured. In the inserts, the Mean Square Displacement (MSD) over a larger set of time intervals is plotted versus the interval length, using a linear (left panel) and logarithmic (right panel) abscissa.

the left panel at small values of the abscissa. However, the exponential 'tail' extends right up to $|\Delta r|=0$ in the glassy regime. Thus, long-ranged spatial correlations survive over long times in dense colloids. This very feature is incorporated as a central hypothesis in the model introduced next.

\section{CLUSTER MODEL}

The cluster model discussed below assumes that strong kinematic constraints bind the particles together inside clusters that form a spatially heterogeneous patchwork of particle properties. The growth and destruction of these clusters are the only mechanisms allowed for the net displacements of particles. The update dynamics is a Markov chain where a randomly selected cluster of (integer) size $h$ either survives intact or is destroyed with probability $P(h)$. By assumption, $0 \leq P(h) \leq 1$ decreases with $h$, i. e. larger clusters are more stable than smaller ones. The particles released by a collapsed cluster join neighboring clusters, and are activated to move in real space by a unit step in a random direction. Model variants are characterized by different forms of $P(h)$. Merely the integrability of $P(h)$ in $h$ discriminates between the behaviors similar to those of low and high density colloids, and without loss of generality we can limit ourselves to

$$
P^{\alpha}(h)=\frac{1}{\sum_{k=0}^{\alpha} \frac{h^{k}}{k !}}, \quad \alpha=1,2 \ldots,
$$

an $\alpha$-family of models with $P^{\alpha=\infty}(h)=\exp (-h)$.

In our Monte Carlo simulations the clusters are arranged in a periodic lattice of length $L$. Initially, each lattice site holds uniformly a cluster of size $h_{i}=\bar{h}$. The av- erage number of particles bound in clusters $\sum_{i=1}^{L} h_{i} / L=$ $\bar{h}$ remains conserved. Note that these clusters do not per-se represent individual colloidal particles and their motion, since particles typically occupy space uniformly, with density fluctuations difficult to discern. Instead, ranges in cluster sizes represent $\mathrm{DH}$ in particle mobilities, which can shift dramatically even though actual particles hardly move. Here, heterogeneities arise $d y$ namically as larger clusters, once formed, are more longlived than smaller ones, and the particles which belong to them correspondingly are less mobile.

We update $t \rightarrow t+1$ in parallel at all even and odd sites in alternating order. Each site $i$ with $h_{i}>0$ is updated by drawing a univariate random number $r_{i}$. If $r_{i}<P^{\alpha}\left(h_{i}\right)$, the cluster on that site breaks up into two randomly split fractions that are respectively added to the neighboring sites $i \pm 1$, leaving $i$ empty. To simulate the actual (activated) motion of particles, we add (mutually non-interacting) tracer particles which reside on lattice sites. Those walk randomly $i \rightarrow i \pm 1$ iff there is a cluster on their site $i$ that happens to break up. In particular, a tracer particle can be stranded on a cluster-free site for a long time until a nearby cluster breaks up such that its scattered "debris" can reactivate that particle.

Fig. 3 shows the MSD averaged over all tracer particles for $\alpha=1$ and $\alpha=\infty$. The former behaves diffusively (up to a cutoff scale $t_{\mathrm{st}} \propto L^{2}$ ). In the latter, no stationary state is approached and the behavior is diffusive in logarithmic time, as in the corresponding experimental result shown in Fig. 1. Fig. 3 also depicts persistence curves, i. e. probabilities that pairs of tracer particles initially located at the same grid point remain within a separation of $\theta$ sites after $t$ sweeps. As in the experiments, the (asymptotic) decay goes from being exponential in time for $\alpha=1$ (not shown [26]) to being exponential in log- 

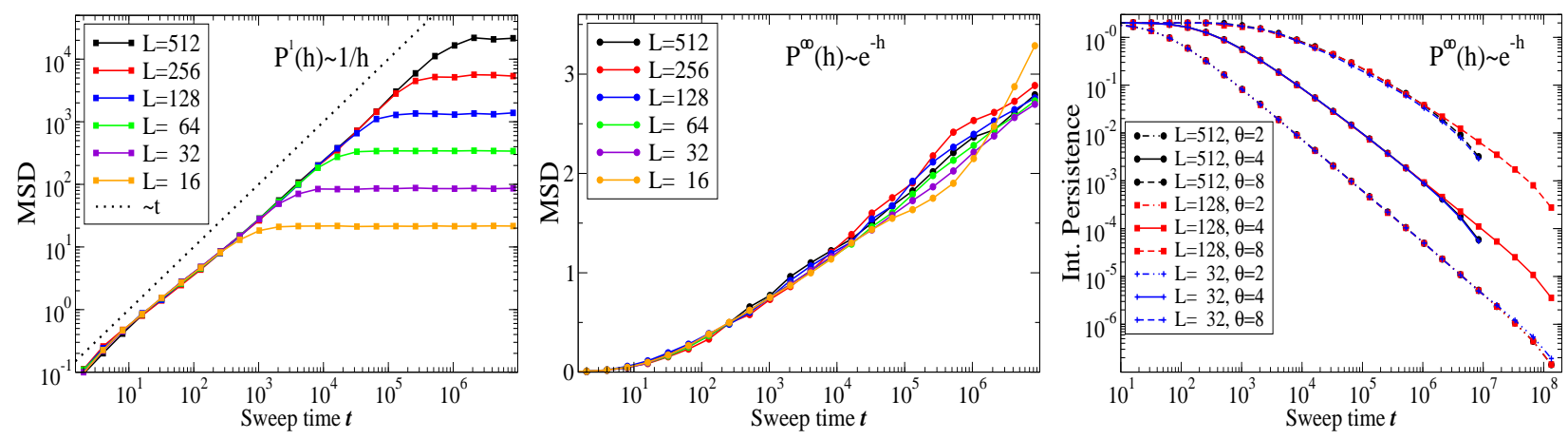

Figure 3: (Color online) Simulation results for different system sizes $L$ for $P^{1}(h) \sim 1 / h$ (left) and $P^{\infty}(h) \sim \exp (-h)($ center and right), to be compared with Fig. 1. For $\alpha=1$ (left) the MSD behaves diffusively, represented by the dotted line of unit slope, while for $\alpha=\infty$ the MSD grows linearly in logarithmic time, with no sign of saturation. The persistence for $\alpha=\infty$ (right) exhibits a power-law, independent of the threshold $\theta$.
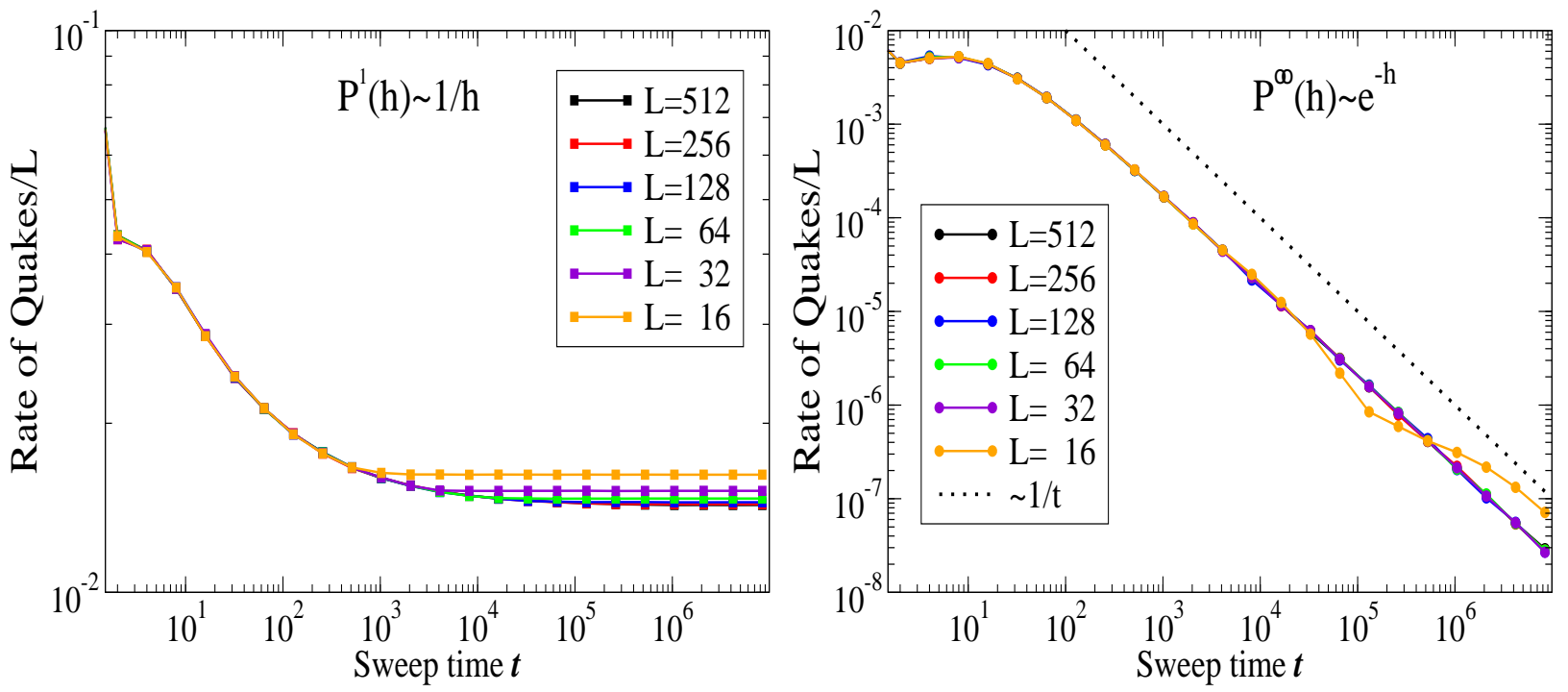

Figure 4: (Color online) Plot of the rate of cluster collapse vs. system age $t$ for $P^{1}(h)$ (left) and $P^{\infty}(h)$ (right).

arithmic time when the cluster collapse probability goes from hyperbolic to exponential at $\alpha=\infty$, independent of $\theta$. Unlike the experiments, changing $\theta$ does not effect the power-law exponent but modifies the time for its onset.

\section{RECORD DYNAMICS}

As the collapse of large clusters controls all aspects of the model in the aging regime, the statistics of such quake-events is key to the analysis, a situation identical to aging in quenched glasses [7]. In turn, the fast collapse of small clusters provides a background of mobile particles diffusing within regions ("cages") bounded by large clusters. The divide between small and large clusters was empirically taken to be $\max (\bar{h}, \sigma(t))$, where $\sigma(t)$ is the (growing) standard deviation of the cluster size distribution. The quake rate versus time, scaled by the system size $L$, is shown in Fig. 4 for our simula- tions. For $P^{1}(h)$ the rate approaches a constant on a time scale independent of system size. For $P^{\infty}(h)$, the stationary regime is in fact invisible even for the smallest systems, and the dynamics is thus purely non-stationary with a decelerating quake rate decaying as $1 / t$. Assuming that successive quakes are statistically independent events (demonstrated elsewhere [26]), they provide the true clock of the dynamics, and the particle MSD simply becomes proportional to the time integral of the quake rate: linear in time in the stationary (liquid) regime, and $\sim \log (t)$ in the aging regime. Clearly then, the MSD in the aging regime between times $t_{w}$ and $t$ scales as $t / t_{w}$, a so-called full aging behavior which is consistent with the experimental findings.

The model dynamics for the exponential collapse probability can be linked to record dynamics [9, 18 21, 27, 28], using the wide separation of time scales associated with the collapse of clusters of different sizes. Let us first note that the empirically estimated boundary between 
small (i.e. irrelevant) and large (i.e. important) clusters, $h(t) \approx \ln t$ can in this case also be obtained by equating to the time $t$ the number of queries that a cluster of height $h$ on average survives. The pace of the dynamics is always controlled by the smallest among the large clusters, henceforth the SL cluster. The latter is the first one to collapses with overwhelmingly high probability. Let the corresponding quake happen on a time scale $t^{(0)}$. The new SL cluster can either be pre-existing or can be formed through the accretion process of the debris produced by the last quake, thus recapitulating all previous history, an accretion process which is now instantaneous on the time scale $t^{(0)}$. Since the cluster size distribution is essentially continuous, the new SL cluster will only be slightly larger than its predecessor, a hallmark of "marginal stability increase" [8]. Hence, a record-sized random number (corresponding to a record sized re-arrangement in the colloid) will suffice to destroy the new SL cluster. At time-step $t$ the probability is $1 / t$ that such event will happen. Statistical independence is assured by the wide separation of break-up events. Thus, inter-event times $\tau^{(n)}=\ln t^{(n)}-\ln t^{(n-1)}=\log \left[t^{(n)} / t^{(n-1)}\right]$ become Poissonian distributed [27, 29]. To ascertain the log-Poisson nature of the quake time distribution, we collect the quake times $t^{(n)}$ and show (elsewhere [26]) that the series $\left(\tau^{(1)}, \tau^{(2)}, \ldots, \tau^{(n)}, \ldots\right)$ of logarithmic waiting times $\tau^{(n)}$ is iid with the same exponential distribution. In contrast, when the cluster collapse probability becomes long-tailed, $\alpha<\infty$, break-up events overlap and longlived clusters are deemphasized.

\section{MEAN-FIELD DESCRIPTION}

Finally, we provide a mean-field model of the clustering dynamics that elucidates the preceding results. The fundamental quantity considered is the average number of clusters $n_{k, t}$ of binary size $h=2^{k}(k=0,1,2, \ldots)$ extant at time $t$. For $k>0$, those $k$-clusters are immobile and break up with probability $p_{k} \sim P\left(2^{k}\right)$, but merely into two $k-1$-clusters. Eq. (11) reduces to $p_{k}^{\alpha} \sim\left(2^{k}\right)^{-\alpha}$. Integrability of $p_{k}$ is conferred via $P(h) d h \sim p_{k} d\left(2^{k}\right) \propto$ $2^{k} p_{k} d k$, i. e. $\sum_{k} 2^{k} p_{k}^{\alpha}<\infty$ for $\alpha>1$. To write down a self-consistent equation for $n_{k, t}$, we stipulate that during each sweep the number of $k$-clusters $n_{k, t}$ for all $k>0$ decreases through break-ups by a factor $1-p_{k}$, yet, it also increases through the break-up of $k+1$-clusters into two $k$-clusters (with weight $\left.2 p_{k+1}\right)$. Unit $(k=0)$-clusters are indivisible but mobile. Larger $k$-clusters further grow via accretion of those small mobile clusters, but (somewhat artificially) only in groups of $2^{k}$ with probability $\propto 1 / 2^{k}$. During a sweep, all unit clusters attach somewhere, i. e. $n_{0, t+1}$ is entirely unrelated to $n_{0, t}$. Hence, we obtain

$n_{k, t+1}=n_{k, t}\left(1-p_{k}\right)+2 p_{k+1} n_{k+1, t}+\frac{n_{k-1, t} n_{0, t}}{2^{k} N_{t}}$

for $k>0$, and $n_{0, t+1}=2 p_{1} n_{1, t}$. The last term describes growth through the accretion of all unit clusters $n_{0, t}$, equally shared between all clusters, $N_{t}=\sum_{k^{\prime}=0}^{\infty} n_{k^{\prime}, t}$. Its form ensures the conservation of $\sum_{k^{\prime}=0}^{\infty} 2^{k^{\prime}} n_{k^{\prime}, t}(=\bar{h} L)$, as used in our simulations above.

Defining $a_{k, t}=2^{k} p_{k} n_{k, t}$, we rewrite Eq. (2) as

$\frac{1}{p_{k}}\left(a_{k, t+1}-a_{k, t}\right)=a_{k+1, t}-a_{k, t}+a_{k-1, t} \mathcal{K}_{k-1} \mathcal{T}_{t}$.

Amazingly, our analysis finds that one of the factors, $\mathcal{K}_{k}=1 /\left(2^{k} p_{k}\right)$ or $\mathcal{T}_{t}=n_{0, t} / N_{t}$, is always sufficiently small asymptotically as to ignore the last term of Eq. (3). The remaining linear equation has stationary solutions (for $t \sim t+1 \rightarrow \infty$ ) entailing $a_{k+1} \sim a_{k} \sim$ const, i. e. $n_{k} \sim 1 /\left(2^{k} p_{k}\right)$. Since the stationary cluster size distribution must have a finite integral (after all, $N_{t} \leq L<\infty$ ), $n_{k}$ must decrease sufficiently, demanding $p_{k} \gg 2^{-k}$ or $\alpha<1$. In this regime, $\mathcal{T}_{t} \rightarrow \mathcal{T}_{\infty} \leq 1$ but $a_{k-1} \mathcal{K}_{k-1} \ll a_{k}$ justifies dismissal of the last term in Eq. (3). Conversely, for $2^{k} p_{k} \ll 1(\alpha>1)$, the aging regime, numerics suggests that $n_{k, t}$ is highly localized at some increasing $k=k(t)$ : clusters grow and attain a typical size $h(t)$. Unit-size clusters hardly ever occur, $n_{0, t}=0$ almost always, and although $\mathcal{K}_{k}$ grows exponentially, $\mathcal{T}_{t} \approx 0$ annihilates the last term. Rescaling $\tau=p_{k} t$ then turns Eq. (3) into a "wave" equation, $\left[\partial_{\tau}-\partial_{k}\right] a(k, \tau) \approx 0$, with characteristic $k \sim \tau=p_{k} t$. Using $p_{k}=p_{k}^{\alpha}$ and $h \sim 2^{k}$, we extract the dominant growth-law for cluster sizes, $h(t) \sim t^{1 / \alpha}$, or for the event-rates (quakes) of cluster-size increases:

$$
\frac{\partial h}{\partial t} \sim t^{\frac{1}{\alpha}-1}, \quad(\alpha>1) .
$$

The scaling reproduces for $\alpha \rightarrow 1^{+}$and for $\alpha \rightarrow \infty$ the simulation results in Fig. 4 (and, correspondingly, those for any $\alpha$ in-between [26]). While the equations do not describe the displacement of tracer particles explicitly, the discussion above implies that the quake rate sets the scale for spread in time for any observable, be it $h(t)$ or MSD: logarithmic for $\alpha \rightarrow \infty$ and linear (with a cut-off) for $\alpha \leq 1$.

\section{CONCLUSIONS}

Experimental data are analyzed showing that colloidal motion is diffusive in either time or logarithmic time, depending on colloidal density. A model explaining these findings is presented, based on the survival probability of clusters, i. e. highly correlated groups of particles, representing dynamically heterogeneous regions of different mobility: a net displacement of a particle in the model requires the collapse of the cluster to which the particle belongs, i. e. a re-arrangement of (possibly large and increasing) correlation patterns. The survival probability of a cluster increases with its size. Once large clusters obtain the capacity to survive indefinitely, $P(h) \ll 1 / h$ for $h \rightarrow \infty$, non-stationary, aging behavior arises, characterized by intermittency and memory effects. Remarkably, 
the $t / t_{w}$ scaling of the experimental data (full aging) is only achieved in the model in the limit of an exponential $P(h)$, corresponding to the extreme value $\alpha=\infty$ of its parameter. In that sense, tuning a parameter such as $\alpha$, say, by changing the volume fraction $\rho$ in a colloid, does not describe a sharp phase transition. But, presumably, the transition between both extremes occurs over a nearly unobservable interval of $\rho$.
Acknowledgments: We thank Eric Weeks for helpful discussion and access to his data. SB is supported by the U. S. National Science Foundation through grant DMR0812204. PS thanks the Physics department of Emory University for its hospitality and its Emerson Center for financial support.
[1] P. Nordblad, P. Svedlindh, L. Lundgren, and L. Sandlund. Time decay of the remanent magnetization in a CuMn spin glass. Phys. Rev. B, 33:645-648, 1986.

[2] H. Rieger. Non-equilibrium dynamics and aging in the three dimensional Ising spin-glass model. J. Phys. A, 26:L615-L621, 1993.

[3] P. Sibani and H. J. Jensen. Intermittency, aging and extremal fluctuations. Europhys. Lett., 69:563-569, 2005.

[4] L. C. E. Struik. Physical aging in amorphous polymers and other materials. Elsevier Science Ltd, New York, 1978.

[5] W. Kob, F. Sciortino, and P. Tartaglia. Aging as dynamics in configuration space. Europhys. Lett., 49:590-596, 2000.

[6] A. Crisanti and F. Ritort. Intermittency of glassy relaxation and the emergence of a non-equilibrium spontaneous measure in the aging regime. Europhys. Lett., 66:253-259, 2004.

[7] P. Sibani. Linear response in aging glassy systems, intermittency and the Poisson statistics of record fluctuations. Eur. Phys. J. B, 58:483-491, 2007.

[8] P. Sibani and S. Christiansen. Thermal shifts and intermittent linear response of aging systems. Phys. Rev. E, 77:041106, 2008.

[9] S. Christiansen and P. Sibani. Linear response subordination to intermittent energy release in off-equilibrium aging dynamics. New Journal of Physics, 10:033013, 2008.

[10] L. Cipelletti, S. Manley, R. C. Ball, and D. A. Weitz. Universal aging features in the restructuring of fractal colloidal gels. Phys. Rev. Lett., 84:2275-2278, 2000.

[11] D. El Masri, M. Pierno, L. Berthier, and L. Cipelletti. Aging and ultra-slow equilibration in concentrated colloidal hard spheres. J. Phys.: Condens. Matter, 17:S3543, 2005.

[12] E. R. Weeks, J. C. Crocker, A. C. Levitt, A. Schofield, and D.A. Weitz. Three-dimensional direct imaging of structural relaxation near the colloidal glass transition. Science, 287:627-631, 2000.

[13] R. E. Courtland and E. R. Weeks. Direct visualization of ageing in colloidal glasses. J. Phys.: Condens. Matter, 15:S359-S365, 2003.

[14] J. M. Lynch, G. C. Cianci, and E. R. Weeks. Dynamics and structure of an aging binary colloidal gel. Phys. Rev. E, 78:031410, 2007.

[15] R. Candelier, O. Dauchot, and G. Biroli. Building blocks of dynamical heterogeneities in dense granular media. Phys. Rev. Lett., 102:088001, 2009.

[16] L. F. Cugliandolo, J. Kurchan, and L. Peliti. Energy flow, partial equilibration, and effective temperature in systems with slow dynamics. Phys. Rev. E, 55:3898-3914, 1997.
[17] H. E. Castillo, C. Chamon, L. F. Cugliandolo, J. L. Iguain, and M. P. Kennett. Spatially heterogeneous ages in glassy systems. Phys. Rev. B, 68:134442, 2003.

[18] P. Sibani and J. Dall. Log-Poisson statistics and pure aging in glassy systems. Europhys. Lett., 64:8-14, 2003.

[19] P. Anderson, H. J. Jensen, L. P. Oliveira, and P. Sibani. Evolution in complex systems. Complexity, 10:49-56, 2004.

[20] L. P. Oliveira, H. J. Jensen, M. Nicodemi, and P. Sibani. Record dynamics and the observed temperature plateau in the magnetic creep rate of type II superconductors. Phys. Rev. B, 71:104526, 2005.

[21] P. Sibani, G. F. Rodriguez and G. G. Kenning. Intermittent quakes and record dynamics in the thermoremanent magnetization of a spin-glass. Phys. Rev. B, 74:224407, 2006.

[22] W. K. Kegel and A. van Blaaderen. Direct observation of dynamical heterogeneities in colloidal hard-sphere suspensions. Science, 287:290-293, 2000.

[23] P. Mayer, H. Bissig, L. Berthier, L. Cipelletti, J. P. Garrahan, P.Sollich and V. Trappe. Heterogeneous dynamics in coarsening systems. Phys. Rev. Lett., 93:115701, 2004.

[24] D. A. Stariolo and G. Fabricius G Fickian crossover and length scales from two point functions in supercooled liquids. J. Chem. Phys., 125:064505, 2006.

[25] P. Chaudhuri, L. Berthier, and W. Kob Universal nature of particle displacements close to glass and jamming transitions. Phys. Rev. Lett., 99:060604, 2007.

[26] S. Boettcher and P. Sibani, unpublished.

[27] P. Sibani and Peter B. Littlewood. Slow Dynamics from Noise Adaptation. Phys. Rev. Lett., 71:1482-1485, 1993.

[28] P. Sibani and H. J. Jensen. How a spin-glass remembers. memory and rejuvenation from intermittency data: an analysis of temperature shifts. JSTAT, page P10013, 2004.

[29] J. Krug and K. Jain. Breaking records in the evolutionary race. Physica A, 358:1, 2005.

[30] Of course, this observation does not dispute the profound consequences, such as memory effects, that can arise from the breaking of time-translational invariance. 